\title{
Accreting Magnetofluids around a Rotating Compact Object with a Dipolar Magnetic Field
}

\author{
Mahboobeh Shaghaghian \\ Department of Physics, Science and Research Branch, Islamic Azad University, Fars, Iran \\ Young Researchers Club, Science and Research Branch, Islamic Azad University, Fars, Iran \\ E-mail: m.shaghaghianegmail.com
}

\begin{abstract}
The dynamics of an axisymmetric stationary disk of accreting magnetofluid with finite conductivity around a rotating compact object is presented here. Along with Maxwell's equations and generalized Ohm's law, the basic equations governing the motion of a finitely conducting plasma in a curved space-time around a slowly rotating compact object are derived. The finite electrical conductivity is taken into account for the plasma, however, the shear viscous stress is neglected as well as the self-gravity of the disk. In this case, energy dissipation occurs only through the finite resistivity. The magnetic stress takes the place of viscous stress in the standard disk model, and extracts angular momentum from the disk. Indeed, angular momentum transfer is controlled by the global structure of the magnetic field. The accreting plasma in the presence of an external dipole magnetic field gives rise to a current in the azimuthal direction. The azimuthal current produced due to the motion of the magnetofluid generates the magnetic field for the disk. Magnetic lines of force can penetrate the accretion disk owing to the presence of finite resistivity. It has been shown that the dipolar magnetic field structure of the central black hole are modified inside the disk. In fact, the magnetic field lines are pushed outward and are continuous across the disk boundary. It has been demonstrated that the accretion with zero angular momentum and radially falling at infinity acquires angular velocity entirely due to the inertial frame dragging as it approaches the compact object and forms an equilibrium structure of thin disk on the equatorial plane of the central star. The inward flow passing through a sub-Alfvenic region becomes super-Alfvenic to fall into the event horizon.
\end{abstract}

25th Texas Symposium on Relativistic Astrophysics - TEXAS 2010

December 06-10, 2010

Heidelberg, Germany 


\section{Introduction}

Accretion on to compact objects is a natural and powerful mechanism for producing highenergy radiation. Since the process of angular momentum removal operates on slower timescales as compared to free-fall time, the infalling gas with sufficiently high angular momentum can form a disklike structure around a central gravitating body that could be thin or thick depending upon their geometrical shapes. The basic equations governing the dynamics of an axisymmetric stationary magnetofluid disk around a compact object in curved space-time are given by Prasanna et al. (1989). Here, we employ these equations in the space-time curved by a slowly rotating compact object accreting matter from the surrounding non self-gravitating disk treated as a non shear viscous plasma having finite conductivity and bulk viscosity. We have been pursuing the fully general relativistic approach for studying the dynamics of the magnetospheric plasma around a slowly rotating compact object through the analysis of fluid as well as the Maxwell's equations, along with the generalized Ohm's law and an equation of state. Similar to our idea (magnetofluid disk in linearized Kerr geometry) have been performed earlier by Bhaskaran et al. (1990), but the essential difference in our calculations lies in the inclusion of finite conductivity and bulk viscosity, also the non-zero radial inflow velocity for the fluids. Inclusion of a finite resistivity particularly is essential for a disk in absence of shear viscosity to liberate gravitational energy. In studying the relativistic magnetohydrodynamic fluid in the magnetosphere of a Kerr black hole, there speaks of two flows. An inward flow due to the strong gravity and a centrifugally driven outward flow orinigate from the plasma's source with zero poloidal velocity (Takahashi et al. 1990). As mentioned in their discussion, the magnetized flow has critical points at the fast and slow magnetosonic points and at the Alfven point. They demonstrated that the MHD flows must become super-Alfvenic to fall into the event horizon or reach infinity along the magnetic field lines.

\section{Formalism}

We are interested in the relativistic magnetized flows accreted from the source of plasma around a Kerr black hole. In the following, we assume the stationary and axisymmetric magnetosphere $\left(\partial_{t}=\partial_{\varphi}=0\right)$ and ignore its self-gravity. Rapidly rotating black hole is an assumption that is usually employed for example in the jet outflows (Camenzind 1986; Takahashi et al. 1990; Koide et al. 2006). However, in our discussions, assumption of slowly rotating black hole is sufficient. Therefore, we will restrict our analyses to the linearised form of Kerr-metric given by

$$
d s^{2}=\left(1-\frac{2 m}{r}\right) c^{2} d t^{2}-\left(1-\frac{2 m}{r}\right)^{-1} d r^{2}-r^{2}\left(d \theta^{2}+\sin ^{2} \theta d \varphi^{2}\right)+\frac{4 a m}{r} \sin ^{2} \theta d t d \varphi .
$$

The motion of the plasma is governed by the equations of motion on the given background geometry $T_{; j}^{i j}=0$, where $T^{i j}$ is the energy-momentum tensor

$$
T^{i j}=\left(\rho+\frac{\bar{p}}{c^{2}}\right) u^{i} u^{j}-\frac{\bar{p}}{c^{2}} g^{i j}-\frac{1}{4 \pi c^{2}}\left(F_{k}^{i} F^{k j}-\frac{1}{4} g^{i j} F_{k l} F^{k l}\right),
$$

which consists of an imperfect fluid in absence of thermal conductivity with the mass density $\rho$, the gas pressure $p$ (it appears in the pressure variable $\bar{p}$ through $\bar{p}=p-\eta_{b} \Theta$, wherein $\eta_{b}$ being the 
bulk viscosity coefficient and $\Theta=u_{; k}^{k}$, the four-velocity $u^{i}$, and an electromagnetic field tensor $F^{i j}$ satisfying Maxwell's equations

$$
F_{; j}^{i j}=-\frac{4 \pi}{c} J^{i}, \quad \& \quad F_{i j, k}+F_{k i, j}+F_{j k, i}=0 .
$$

The field tensor is defined as $E_{\alpha}=F_{\alpha t}$ and $B_{\alpha}=\varepsilon_{\alpha \beta \gamma} F_{\beta \gamma}$, where $\varepsilon_{\alpha \beta \gamma}$ is the Levi-Civita symbol. The finite conductivity $\sigma$ is taken into account for the plasma through the generalized Ohm's law $J^{i}=\sigma F_{k}^{i} u^{k}$. The Maxwell's equations (Eqs. (2.3)) in the linearised Kerr metric (Eq. (2.1)), may be written as

$$
\begin{aligned}
& \frac{4 \pi}{c} J^{r}=-\frac{1}{r^{2} \sin \theta}\left(1-\frac{2 m}{r}\right) \frac{\partial}{\partial \theta}\left(\sin \theta B_{\varphi}\right) \\
& \frac{4 \pi}{c} J^{\theta}=\frac{1}{r^{2}} \frac{\partial}{\partial r}\left[\left(1-\frac{2 m}{r}\right) B_{\varphi}\right], \\
& \frac{4 \pi}{c} J^{\varphi}=\frac{1}{r^{4} \sin \theta} \frac{\partial}{\partial \theta}\left(\frac{B_{r}}{\sin \theta}\right)-\frac{1}{r^{2} \sin ^{2} \theta} \frac{\partial}{\partial r}\left[\left(1-\frac{2 m}{r}\right) B_{\theta}\right] \\
&-\frac{2 a m}{r^{2}}\left[\frac{\partial}{\partial r}\left(\frac{E_{r}}{r}\right)+\frac{1}{r^{3} \sin \theta}\left(1-\frac{2 m}{r}\right)^{-1} \frac{\partial}{\partial \theta}\left(\sin \theta E_{\theta}\right)\right], \\
& \frac{4 \pi}{c} J^{t}=-\frac{1}{r^{2}} \frac{\partial}{\partial r}\left(r^{2} E_{r}\right)-\frac{1}{r^{2} \sin \theta}\left(1-\frac{2 m}{r}\right)^{-1} \frac{\partial}{\partial \theta}\left(\sin \theta E_{\theta}\right) \\
&-\frac{2 a m}{r^{5} \sin \theta}\left(1-\frac{2 m}{r}\right)^{-1} \frac{\partial}{\partial \theta}\left(\sin \theta B_{r}\right)+\frac{2 a m}{r^{2}} \frac{\partial}{\partial r}\left(\frac{B_{\theta}}{r}\right), \\
& \frac{\partial B_{\theta}}{\partial \theta}+ \frac{\partial B_{r}}{\partial r}=0, \\
& \frac{\partial E_{\theta}}{\partial r}-\frac{\partial E_{r}}{\partial \theta}=0 .
\end{aligned}
$$

In order to simplify the equations, the assumption of vanishing the toroidal component of the electric field $\left(E_{\varphi}=0\right)$ for an axisymmetric fluid seems to be reasonable hypothesis. Also, if the toroidal component of the magnetic field is presumed as $B_{\varphi}=\frac{b_{\varphi}}{\sin \theta}\left(1-\frac{2 m}{r}\right)^{-1}$, then the poloidal current may be vanished $\left(J^{r}=J^{\theta}=0\right)$, where $b_{\varphi}$ is an arbitrary constant. Then, the Ohm's law yields $\left(E_{r}=B_{\theta} \frac{V^{\varphi}}{c} \& E_{\theta}=-B_{r} \frac{V^{\varphi}}{c}\right)$, and the relations for the nonzero current components

$$
\begin{aligned}
& J^{\varphi}=-\sigma B_{\theta} u^{r}\left[\frac{1}{r^{2} \sin ^{2} \theta}+\frac{2 a m}{r^{3}}\left(1-\frac{2 m}{r}\right)^{-1} \frac{V^{\varphi}}{c}\right], \\
& J^{t}=-\sigma B_{\theta} u^{r}\left(1-\frac{2 m}{r}\right)^{-1}\left[\frac{V^{\varphi}}{c}-\frac{2 a m}{r^{3}}\right] .
\end{aligned}
$$

By solving the azimuthal component of momentum equation, rotational velocity is obtained as $V^{\varphi}=\frac{2 a m c}{r^{3}}$. This velocity is perfectly due to the inertial frame dragging induced by the space-time surrounding the rotating compact object (Prasanna 1989). This is entirely the general relativistic feature of space-time physics and indeed such solution doesn't exist in Newtonian theory. Consequently, the azimuthal velocity doesn't find the Keplerian distribution in the limit of the classical 
case. Furthermore, once pressure provides substantial support in the radial direction, the angular momentum distribution may be far from Keplerian. To appreciate the effects of rotation of the central compact object on the disk dynamics, translate the equations into the frame as seen by the locally non-rotating observer outside a rotating black hole (Bardeen et al. 1972)

$$
\begin{aligned}
& \frac{\partial}{\partial r}\left[r^{2} B_{(r)}\right]+\frac{r}{\sin \theta}\left(1-\frac{2 m}{r}\right)^{-1 / 2} \frac{\partial}{\partial \theta}\left[\sin \theta B_{(\theta)}\right]=0, \\
& \frac{\partial}{\partial r}\left[\frac{B_{(r)}}{r}\right]+\frac{1}{r^{2} \sin \theta}\left(1-\frac{2 m}{r}\right)^{-1 / 2} \frac{\partial}{\partial \theta}\left[\sin \theta B_{(\theta)}\right]=0 .
\end{aligned}
$$

One admissible solution set for the magnetic field given by the Eq. (2.12) is

$$
B_{(r)}=-B_{1} r^{k} \sin ^{k} \theta \cos \theta, \quad \& \quad B_{(\theta)}=B_{1} r^{k}\left(1-\frac{2 m}{r}\right)^{1 / 2} \sin ^{k+1} \theta
$$

The magnetic field is generally decomposed into two parts; the seed field $\vec{B}^{S}$ caused by some external sources and the disk field $\vec{B}^{D}$ caused by the current flowing in the accretion disk $\vec{B}=$ $\vec{B}^{S}+\vec{B}^{D}$. The external magnetic field $\vec{B}^{S}$ is considered to be dipolar that fits for a modelling of the accretion disks around compact stars in close binary systems. The central star is assumed to be a black hole which can't generate the magnetic field itself. But, the current streaming outside the event horizon can make an external magnetic field around the black hole. Form of the dipole magnetic field around a rotating black hole has been investigated by some authors (Prasanna 1978; Prasanna \& Vishveshwara 1978; Takahashi \& Koyama 2009)

$$
\begin{aligned}
& B_{r}^{S}=-\frac{3 \mu}{4 m^{3}} r^{2}\left\{\frac{2 m}{r}\left(1+\frac{m}{r}\right)+\ln \left(1-\frac{2 m}{r}\right)\right\} \sin \theta \cos \theta, \\
& B_{\theta}^{S}=\frac{3 \mu}{4 m^{2}}\left\{1+\left(1-\frac{2 m}{r}\right)^{-1}+\frac{r}{m} \ln \left(1-\frac{2 m}{r}\right)\right\} \sin ^{2} \theta,
\end{aligned}
$$

wherein $\mu$ is the dipole magnetic moment of the central star that may be expressed in terms of the surface magnetic field $B_{s}$ and the radius $R$ of the central object as $\mu=B_{s} R^{3}$.

\subsection{Magnetic Field Configuration}

To study the magnetic field configuration, we look at the magnetic field lines which satisfy the relation $\frac{d r}{B_{(r)}}=\frac{r d \theta}{B_{(\theta)}}=\frac{r \sin \theta d \varphi}{B_{(\varphi)}}$. In order to visualize the field line configuration, it is useful to express the results in a Cartesian frame through the usual relations $(X=r \sin \theta \cos \varphi, Y=r \sin \theta \sin \varphi$ and $Z=r \cos \theta$ ). Applying these transformations, the dipolar configuration of the external magnetic field at infinity

and the disk magnetic field configuration

$$
X=\frac{r_{0}}{\sin \theta}, \quad \& \quad Z=\frac{r_{0}}{\sin ^{2} \theta} \cos \theta,
$$

$$
X=r_{0} \cos \left(\varphi_{0}-\beta r_{0}^{-(k+1)} \cot \theta\right), \quad \& \quad Y=r_{0} \sin \left(\varphi_{0}-\beta r_{0}^{-(k+1)} \cot \theta\right), \quad \& \quad Z=r_{0} \cot \theta,
$$

are achieved. Here, $\varphi_{0}$ is a constant of integration and $\beta=\frac{b_{\varphi}}{B_{1}}$. Since, we consider axisymmetric solutions, we can set $\varphi_{0}$ to zero without any loss of generality. Fig. 1 shows a typical profile of the magnetic field structure in the meridional plane without (Fig. 1a) and with the disk field (Fig. 1b). 

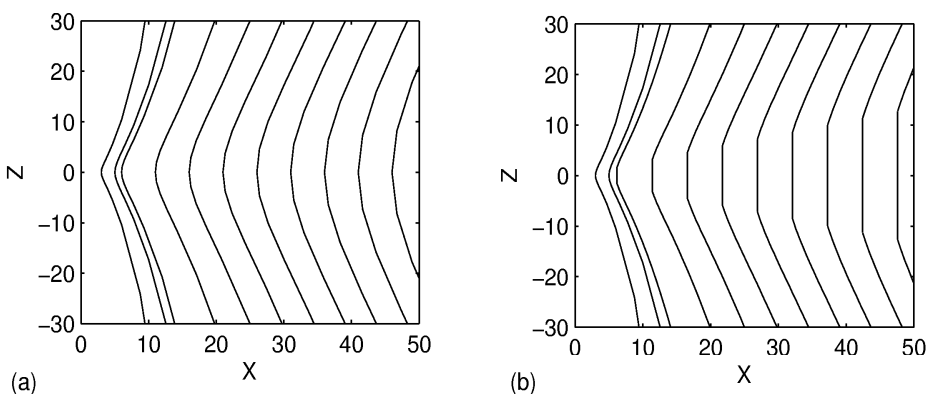

Figure 1: Structure of magnetic field lines a) without the disk b) with the disk, for $\beta=0$.

For the purpose of illustration, the meridional structure of the disk is presumed to extend to about $15^{\circ}$ on either side of the equatorial plane. It is seen on comparison that, inside the disk, the field lines are pushed outward as the parallel to the $z$ axis. It is clear that the constant field lines of the disk are connected with the lines of the external field at the surface of the disk and are continuous at the boundary. If the plasma disk is considered to be infinitely conducting, any external magnetic field can not penetrate the disk. However, as pointed out by Ghosh \& Lamb (1978), magnetic lines of force can penetrate the accretion disk owing to finite resistivity.

\section{Possible equilibrium solutions}

\subsection{Special Case $\sigma=0$}

As the first step in way of solving the equations and investigating the system's behavior, we are interested in the special case of non-conducting plasma. In order to balance the number of equations with the number of unknown variables $\left(\rho, \bar{p}\right.$ and $\left.V^{(r)}\right)$, it is necessary to include a state equation. If we define a new variable for density as $\bar{\rho}=\rho-\frac{\bar{p}}{c^{2}}$, we can consider the state equation of constant density as $\bar{\rho}=\rho_{0}$, (in case $\rho_{0}$ is constant). Applying the thin disk approximation $\left(\theta=\frac{\pi}{2}\right.$ and $V^{\theta}=0$ ), the radial component of motion equation and the continuity equation are simplified respectively as

$$
\begin{aligned}
& \frac{d}{d r} \ln \left(2 \rho-\rho_{0}\right)=-\frac{2}{c^{2}}\left(1-\frac{\left(V^{(r)}\right)^{2}}{c^{2}}\right)^{-1}\left[V^{(r)} \frac{d V^{(r)}}{d r}+\frac{m c^{2}}{r^{2}}\left(1-\frac{2 m}{r}\right)^{-1}\left(1-\frac{\left(V^{(r)}\right)^{2}}{c^{2}}\right)\right] \\
& \frac{d}{d r} \ln V^{(r)}=-\frac{2}{r}-\frac{6 a m}{r^{2} c}\left(1-\frac{2 m}{r}\right)^{-1} V^{\varphi}
\end{aligned}
$$

Integrating them yields

$V^{(r)}=n c \frac{m^{2}}{r^{2}}\left(1-\frac{2 m}{r}\right)^{-\frac{3 a^{2}}{4 m^{2}}} e^{-\frac{3 a^{2}}{2 m^{2}}\left(\frac{m}{r}+\frac{m^{2}}{r^{2}}+\frac{4 m^{3}}{3 r^{3}}\right)}, \quad \& \quad \rho=\rho_{0}\left(1-\frac{2 m}{r}\right)^{-1}\left(1-\frac{\left(V^{(r)}\right)^{2}}{c^{2}}\right)$,

where $n$ and $\rho_{0}$ are the constants of integration. Then, the gas pressure is achieved as

$$
p=\bar{p}+\eta_{b} \Theta=\left(\rho-\rho_{0}\right) c^{2}+\eta_{h} \Theta,
$$




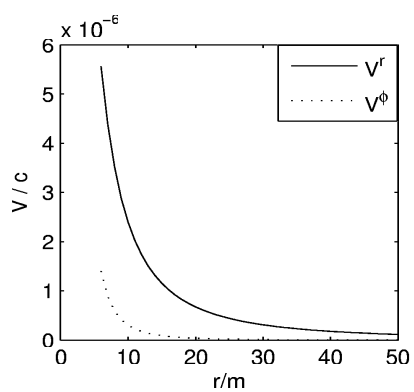

Figure 2: Profile of velocities as functions of radial distance in unit of mass of the black hole $(\mathrm{r} / \mathrm{m})$ with $\sigma=0, \alpha=0.9, n=3 \times 10^{-4}$. The radial velocity (solid line) as well as the azimuthal velocity (dotted line) measured in BLF.

wherein $\Theta=u_{; k}^{k}=u_{, r}^{r}+\frac{2}{r} u^{r}$. In this case, there are no electromagnetic fields, subsequently, no magnetic stresses. In other words, in this case, there isn't any angular momentum transport mechanism. Thus, these solutions are justified as the accretion flow in final stages once most of the gas orbital angular momentum already has been removed in the sense that in those instants the fluids almost pass directly into the central compact object (Ghanbari \& Shaghaghian 2009). Close to the black hole event horizon, the gas temperature and velocities become extremely high (Popham \& Gammie 1998) and gradually fall off outwards. As shown in Fig. 2, due to the condition of accretion flow in last stages, the fluids' radial inflow velocity is several times faster than its rotation. On account of this reason, there exists a lower bound on parameter $n$.

\subsection{General Case $\sigma \neq 0$}

Since the solution of equations in presence of conductivity should get the previous ones (Eqs. (3.3)) in case $\sigma=0$, we presume the solutions as

$$
\begin{aligned}
V^{(r)} & =n c\left[\frac{m^{2}}{r^{2}}\left(1-\frac{2 m}{r}\right)^{-\frac{3 a^{2}}{4 m^{2}}}-\frac{\sigma m}{c} f\left(\frac{r}{m}\right)\right] e^{-\frac{3 a^{2}}{2 m^{2}}\left(\frac{m}{r}+\frac{m^{2}}{r^{2}}+\frac{4 m^{3}}{3 r^{3}}\right)}, \\
\rho & =\rho_{0}\left(1-\frac{2 m}{r}\right)^{-1}\left[1-n^{2} \frac{m^{4}}{r^{4}}\left(1-\frac{2 m}{r}\right)^{-\frac{3 a^{2}}{2 m^{2}}} e^{-\frac{3 a^{2}}{m^{2}}\left(\frac{m}{r}+\frac{m^{2}}{r^{2}}+\frac{4 m^{3}}{3 r^{3}}\right)}+\frac{\sigma m}{c} g\left(\frac{r}{m}\right)\right],
\end{aligned}
$$

where $f$ and $g$ are the unknown dimensionless functions. Consistency of Eq. (2.10) with the Maxwell's equation (Eq. (2.6)) leads us to an exact solution for the radial inflow velocity in LNRF given by

$$
V^{(r)}=c\left(1+\left(\frac{4 \pi \sigma}{c k}\right)^{2} r^{2}\left(1-\frac{2 m}{r}\right)^{-1}\right)^{-1 / 2} .
$$

Fig. 3a gives the profile of the poloidal velocity in comparison with the Alfven velocity for two values of $\sigma$. As expected, the radial inflow is sub-Alfvenic in the outer region, and after passing an Alfven point (i.e. the point where the inflow velocity reaches the Alfven velocity), becomes superAlfvenic in the inner region. Because of this fact, there exists a lower bound on the density free 

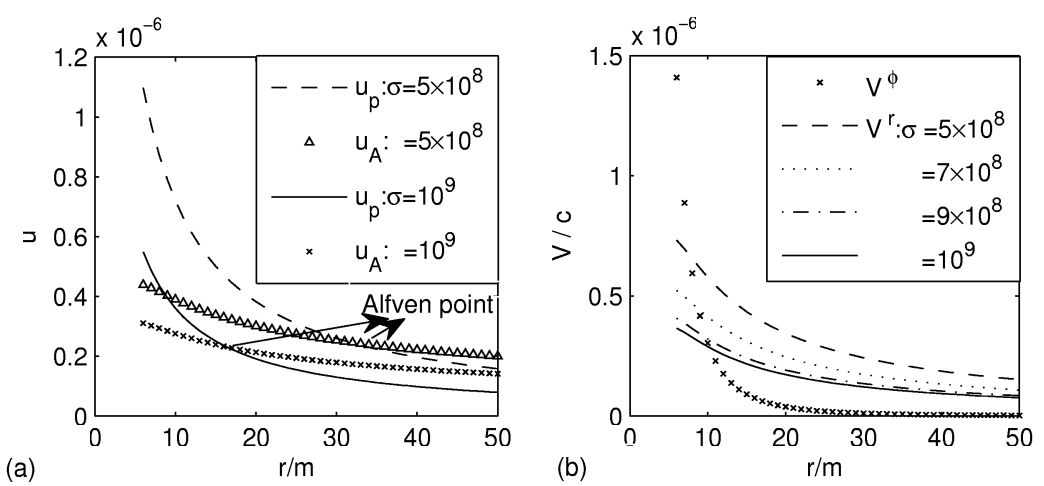

Figure 3: Profiles of velocities. (a) The poloidal velocity and the Alfven velocity for two values of $\sigma$ represented in legend. (b) $(x \times \times)$ indicates the azimuthal velocity and the other lines are the radial velocity (in BLF) for different values of $\sigma$ represented in legend. The constant parameters are as follows: $\alpha=$ $0.9, n=1, k=1, \rho_{0}=0.4 \times 10^{7} \mathrm{~kg} / \mathrm{m}^{3}$ and $B_{S}=10^{8} \mathrm{~T}$.

parameter $\rho_{0}$ below which the Alfven velocity becomes so fast in the sense that the accretion flow becomes sub-Alfvenic in whole regions. The conductivity consideration gives rise to the presence of the magnetic field in the governing equations. Therefore, in this case, the magnetic stresses play the role of angular momentum transport mechanism. Accordingly, these solutions can be indicated the accretion flow in the middle stages, once the radial and the azimuthal velocities of the plasma are of the same order (Fig. 3b). Fig. 3b shows that in the outer regions, the fluids' radial inflow is done faster than its rotation. However, gradually toward the inner regions, the rotation becomes faster than the inflow. As $\sigma$ becomes larger, both the radial inflow velocity and the Alfven velocity slow down. The Alfven point becomes nearer to the inner boundary and the vastness of the superAlfvenic region reduces (Fig. 3a).

\section{References}

[1] J.M. Bardeen W.H. Press and S.A. Teukolsky, 1972, ApJ, 178, 347

[2] P. Bhaskaran S.C. Tripathy and A.R. Prasanna, 1990, JApA, 11, 461

「31 M. Camenzind, 1986, A\&A, 162, 32

[4] J. Ghanbari and M. Shaghaghian, 2009, PASJ, 61, 1261

[5] P. Ghosh and F.K. Lamb, 1978, ApJ, 223, L83

「6] S. Koide K. Takahiro and S. Kazunari, 2006, PhRvD, 74, 4500

[7] R. Popham C.F. Gammie, 1998, ApJ, 504, 419

「81 A.R. Prasanna, 1978, BASI, 6, 88

[9] A.R. Prasanna and C.V. Vishveshwara, 1978, Pramana, 11, 359

[10] A.R. Prasanna, 1989, A\&A, 217, 329

[11] A.R. Prasanna S.C. Tripathy and A.C. Das, 1989, JApA, 10, 21

[12] M. Takahashi S. Nitta Y. Tatematsu and A. Tomimatsu, 1990, ApJ, 363, 206

[13] M. Takahashi and H. Koyama, 2009, ApJ, 693, 472 